\title{
29
}

\section{The MAPS control paradigm: using chaotic maps to control telecoms networks}

L.G. Samuel, J.M. Pitts, R.J. Mondragón, D.K. Arrowsmith Queen Mary and Westfield College Mile End Road, London E1 4NS, United Kingdom, e-mail: \{L.Samuel, J.M.Pitts, R.J.Mondragon, D.K.Arrowsmith\} @qmw.ac.uk Tel: +44-171-415-3756 Fax:+44-181-981-0259

\begin{abstract}
This paper proposes a new control method for telecommunications networks based on chaotic dynamics. Self-similar behaviour has been observed in a variety of services and networks. ATM is a unifying vehicle for transporting a wide range of traffic streams, and hence is a focus for assessing the impact of self similarity on networks. Chaotic maps have been used to model self-similar traffic. Using this approach, we present results that show the sensitivity of the Hurst parameter to the dynamical parameters. Conventional control techniques for ATM each address only a single time scale, and hence are less effective as a result of self-similarity in the traffic behaviour. We set out the MAPS control paradigm, using local control techniques in coupled map lattices, which aims to effect control over the range of relevant timescales.
\end{abstract}

\section{Keywords}

Self-similar traffic, ATM networks, chaotic map, chaotic control 


\section{INTRODUCTION}

ATM is becoming the transport vehicle for a wide variety of traffic streams, whether it is legacy traffic, LAN-LAN, Internet IP, multimedia, etc. ATM was created as a unifying transport mechanism. The mechanism provides the means to statistically multiplex variable and constant bit rate streams. One of the main features of ATM is its statistical multiplexing gain (Saito, 1994). Statistical multiplexing gain arrives out of multiplexing traffic streams where the sum of the individual peak bandwidths is greater than the capacity of a given link (Chen, 1995). This is possible because the peaks in the individual traffic streams seldom occur together. Therefore the statistical multiplexing effect relies on the condition that enough sources are multiplexed and that they are not correlated (de Prycker, 1991). The analysis of such a gain has been attempted under the assumptions of Poisson arrival processes and exponential distributed holding times (Saito, 1994). The implication of this type of analysis is that such traffic streams when aggregated tend to white Gaussian noise, i.e. the variation of the traffic would eventually smooth out (see Figure.1 after Figure 4 in Leland 1994). However, traffic measurements carried out in the late 1980's and early 1990's revealed that whereas the correlations of the traffic were thought to decay exponentially fast (Markovian in structure) the traffic measured in real networks possessed correlation structures which decayed much slower than exponentially (Fowler, 1991). This type of traffic has become known as Long Range Dependent (LRD).

Why should this be a problem? The answer lies in the correlation structure of the LRD traffic. Heuristically, one can view the individual traffic streams' correlation as overhanging each other when aggregated, causing an increase in the probability of the large aggregated bursts occurring. More importantly the aggregated traffic streams do not tend to white Gaussian noise (for a representation of these effects see Figure 1). In actual fact the aggregated traffic process tends towards a secondorder statistically self-similar process which remains bursty over many time scales (Fowler, 1991). This feature of the traffic poses problems for the traffic control schemes designed for ATM. In ATM preventative congestion control is preferred over reactive congestion control schemes. This is because the reactive control becomes inadequate in terms of response times for the high bit rates used in ATM (Chen, 1995). The preventative measures are concentrated in the connection admission control schemes (CAC) used to make decisions on the acceptance of calls into the system. It now appears that CAC cannot minimise the congestion within the network and increasing buffer sizes also appear to have no effect (Leland, 1994). For this reason the time has arrived to consider control schemes which are based on totally different paradigms. In this paper we present such a paradigm based on chaotic dynamics.

The organisation of the paper is as follows: in section 2 we give a brief introduction on the nature and effects of self-similar traffic; in section 3 we outline 
how dynamical systems can be used to model teletraffic; and in section 4 we describe the principles of chaotic control.

\section{Real Traffic}
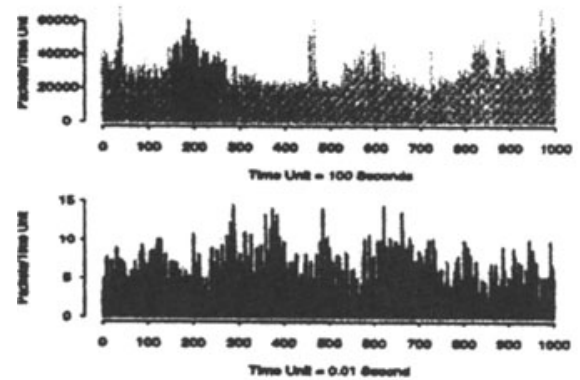

Markov Models
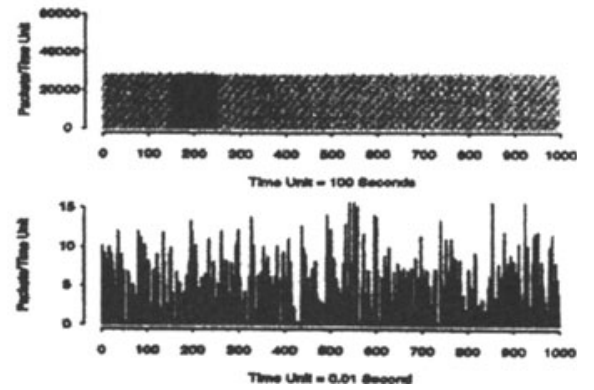

Figure 1 Real traffic trace against Markov model based trace for the same load. (The picture is taken from figure 4 in Leland (1994), Reproduced with permission).

\section{THE IMPACT OF SELF-SIMILAR TRAFFIC}

Fowler (1991) reported on studies conducted at the at the end of the 1980's and early 1990's that packet traffic exhibited burstiness over a large number of time scales. These bursts exist at every time scale, from milliseconds to days and they look similar independently of the time scale, i.e. the traffic is self similar. One characteristic of this self-similar traffic is that it is correlated at all time scales of engineering interest, i.e. the traffic has long range dependence (LRD). The selfsimilarity and the LRD are quantified by the Hurst parameter $H(1 / 2 \leq H<1)$. Large values of $H$ correspond to larger fluctuations on the burst size and stronger correlations in the traffic. These large fluctuations manifest themselves as heavy tailed distribution in the LRD traffic (Leland, 1994) and has been linked to the probability of higher buffer occupancy (Norros, 1993). Practically this increased probability has a drastic effect on the buffer occupancy since providing more buffer space is not a solution to buffer saturation (Eramilli, 1996). Eventually the buffer will fill up. The implication that an increasing value of $H$ leads to higher buffer state occupancy (Norros, 1993, Eramilli, 1996) and hence increased probability of network congestion is much higher than if low valued $H$ traffic was present which is interesting from the point of developing a chaotic network control since a small adjustments in $H$ can drastically alter the buffer occupancy.

A natural question to ask would be: if CAC is conservative (in the sense of admitting traffic to suit the bottle neck link) then why is it that cell loss still occurs? The answer to this question lies in results presented by Willinger (1997). They have shown that it is the aggregation of $O N-O F F$ sources with $O N$ and/or $O F F$ periods which are long range dependent that causes the self-similarity present 
in the networks. It is the self-similarity and the LRD of the aggregated traffic which is perceived as the future cause of network congestion (Leland 1994, Fowler, 1991, Erramilli 1996). A first step in finding solutions to the problems caused by self-similar traffic was research on models which adequately capture the variability seen in real traffic. Conventional stochastic traffic models, based in Markovian traffic theory, describe real traffic only over a single timescale. They do not have LRD (see Figure 1). There exist alternative stochastic traffic models known as Fractional Brownian Motion (FBM) models (Mandelbrot, 1968, Norros, 1993) which describe all the traffic characteristics of real traffic, the self-similarity and LRD. Alternatively, there exist models based on chaotic maps which reproduce the properties of real traffic (Eramilli 1994a, Samuel, 1997b). The need for the new models was so that the impact of self-similar behaviour could be assessed against current and proposed provisioning practices for ATM networks. Broadly, this research has taken the form of assessing the impact on the buffers due to the self-similar traffic. The motivation for this research could be said to be an attempt to reconcile the control of this type of traffic into some queuing theoretic approach, which can then be used in "traditional ways" in order to solve network provisioning and CAC (in ATM terms) problems that arise. In the following paragraphs these approaches will be briefly outlined.

Certain approaches have been traffic class specific. For example traffic classes such as $\mathrm{VBR}_{\mathrm{RT}}$ and $\mathrm{CBR}_{\mathrm{RT}}$ are very delay sensitive and have used control paradigms based on buffer partitioning (Schormans, 1997) and priority scheduling (Schormans, 1993) to solve the congestion. That is to say, the play-out rate of the occupied portion of the buffer will be sufficiently fast enough as to make the Markovian model estimation of the resource requirement conservative enough to be useful.

One approach which one would have naturally thought that would have at least reduced the impact of self-similar traffic on the network would have been traffic shaping. One would have thought that spreading the burstiness of the individual traffic sources would have altered the characteristics of the traffic sufficiently to the point where individual traffic streams do not become a problem. Unfortunately this is not the case (Leland, 1994). Work undertaken recently by Molonár (1997) shows that shaping will not alter greatly the self-similarity present in the traffic. A robust indication of this could be implied for the work of Eramilli (1996) where experiments on reshuffled LRD data were undertaken. Essentially the entire order of a data stream had to be shuffled randomly before the LRD nature in the strearn was lost. If all the LRD streams are shaped then all that is achieved is an extension over the period over which the self similar traffic is present. This is because shaping still preserves the order of the data.

Another approach had been to accept Markovian models as adequate and use large deviation techniques to asses the impact of the rare event "large bursts" on a queueing system. Here, large deviation theory is used to calculate the probability of buffer overflow. This information is then used to provision the network/accept 
calls accordingly. This too has led to the formulation of CAC algorithms based on this principle (Duffield, 1995).

Naturally there have been approaches which combine FBM modelling and large deviations theory in order to arrive at some qualitative assessment of the effects self-similar traffic has on network buffering systems. These approaches have led to the notion of cross-over effects when self-similar streams are multiplexed together (Krishnan, 1996 and Fan, 1997). This effect describes an increase in the multiplexing gain in the buffering system when streams of self-similar traffic are multiplexed. However, once beyond the cross-over point the self-similar traffic streams once again are detrimental to the network. The point in this approach is however, that Markovian models (those with self-similarity parameter $H=0.5$ ) provide good (conservative) estimates for the buffer sizes required by the system in order to cope with the self-similar traffic streams.

Having summarised very briefly the traditional (current) control methods we now look comparatively at the way in which control affects common areas where chaotic control could be applied. The control of network traffic could be viewed as:

1. Call level control - permits the traffic onto the network provided that there are enough resources on the requested path which permit the incoming traffic to propagate across the network with out causing congestion at the cell level.

2. Cell level control - allocates resources in the network (such as buffer space) in order to accommodate the call in terms of cells or allow some traffic loss according to some pre-agreed cell loss rate.

Both (1) and (2) have traditionally been handled on entry onto the network via $\mathrm{CAC}$ algorithms. Additionally (2) can be approached via the design/dimensioning of the switches and cell level control methods such as UPC. In MAPS control, call level control is termed "order". This is the selection of call or burst based on a weighted decision derived form the dynamics of the system (network). Cell level control is termed "procession" and is the effect of the control of the dynamical system which permits the transfer of the data between source and sink.

\section{DYNAMICAL SYSTEMS APPROACH TO TELETRAFFIC}

An ON/OFF traffic source can be modelled using the family of one dimensional chaotic maps (Erramilli, 1994a, Erramilli, 1994b, Pruthi 1995a)

$$
x_{n+1}=F\left(x_{n}\right)= \begin{cases}F_{1}\left(x_{n}\right)=\varepsilon_{1}+x_{n}+x_{n}^{m_{1}}\left(1-\varepsilon_{-}-d\right) / d^{m_{1}}, & 0<x_{n} \leq d \\ F_{2}\left(x_{n}\right)=\varepsilon_{2}+x_{n}+\left(1-x_{n}\right)^{m_{2}}\left(\varepsilon_{2}-d\right) /(1-d)^{m_{2}}, & d<x_{n}<1\end{cases}
$$


with parameters $m_{1}, m_{2} \in(3 / 2,2), \varepsilon_{1}, \varepsilon_{2} \ll 1$ and $0<d<1$. The ON-OFF traffic source is simulated by the associated indicator random variable (see Figure 2)

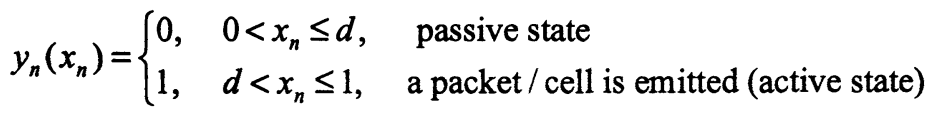

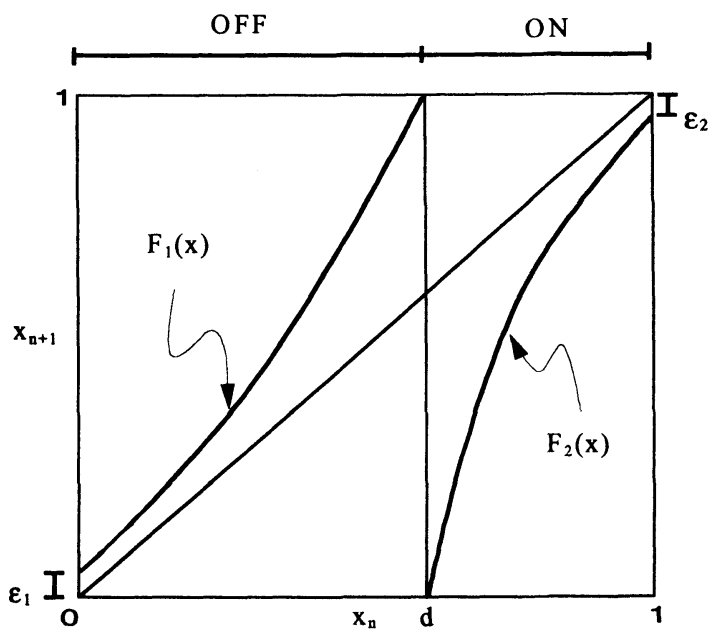

Figure 2 Chaotic Intermittency map showing parameters $d, \varepsilon_{l}$ and $\varepsilon_{2}$ and curve functions $\mathrm{F}_{1}(\mathrm{x})$ and $\mathrm{F}_{2}(\mathrm{x})\left(m_{1}\right.$ and $m_{2}$ describe the degree of polynomial for $\mathrm{F}_{1}(\mathrm{x})$ and $F_{2}(x)$ respectively).

In common with measured traffic, the traffic simulated by these maps has a noninteger fractal dimension $(1 / 2 \leq H<1)$, long range dependence (LRD) on the correlation and, if the outputs of several maps are aggregated, the simulated traffic tends to FBM. For a summary of the different map interpretations as a source model the interested reader is referred to the following references (Eramilli,1994a and 1994b, Pruthi, 1995b, Samuel, 1997a and1997b).

Traffic with different characteristics is labelled by the parameters of the maps. However, from a control point of view it is better to study the family of maps rather than any individual member of the family. To this end the Bernoulli shift map (which models Poisson-like behaviour, $m_{1}=m_{2}=1$ ), the single intermittency map (which models traffic with LRD in either $O N$ or $O F F$ state, $m_{1}=1, m_{2}>3 / 2$ or $m_{1}>3 / 2, m_{2}=1$ ) and the double intermittency map (which models traffic with long range dependence in both $O N$ and $O F F$ states $m_{1}>3 / 2, m_{2}>3 / 2$ ) are all considered members of the same family. These different traffic models are consistent with the reported characteristics of $O N-O F F$ sources by Willinger (1997). 
As a first stage in developing a chaotic control for telecommunication networks we have studied how $H$ changes with the map parameters. A decision to alter $H$ based on the adjustment of these parameters can be interpreted as the controlling action. For example an alteration in the value of $\varepsilon$ imposes an upper cut-off on the correlation, alteration in $d$ changes the mean traffic load and changes in $m_{1}$ and $m_{2}$ changes the sojourn time of the $O N$ and $O F F$ states, i.e. the LRD of the traffic and its $H$ value. Figure 3 shows two examples of the dependence of $H$ with the map parameters.

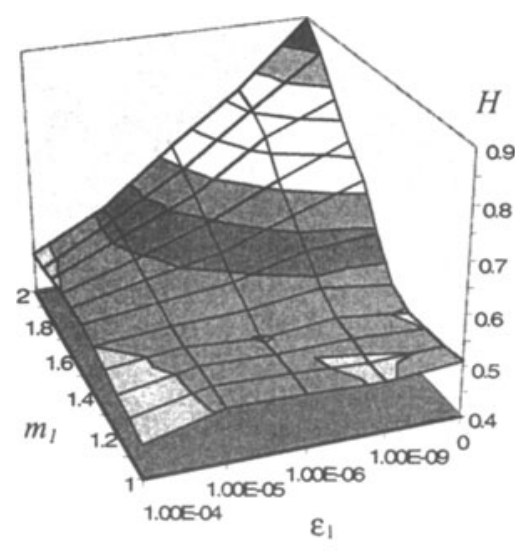

(a)

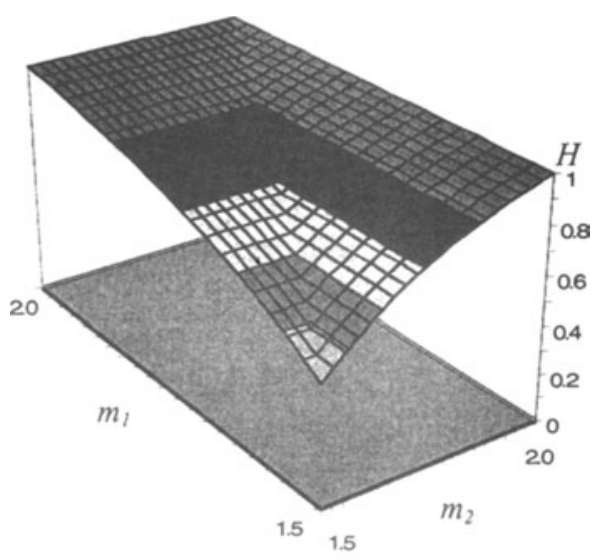

(b)

Figure 3 The dependence of the Hurst parameter on (a) $m_{l}$ and $\varepsilon_{l}$, and (b) $m_{l}$ and $m_{2}$. All other parameters are fixed.

Figure 3(a) shows that, for a single intermittency map, as $\varepsilon_{l}$ increases, the value of $H$ tends to 0.5 and the long range dependence disappears. Figure 3(b) shows that, for a double intermittency map, the value of $H$ depends on both $m_{l}$ and $m_{2}$. These results show Eramilli's conjecture (Eramilli, 1995, conjecture 3 ) that $H$ is a function only of $m_{l}$ to be false.

As all sources can be modelled by a map, the next stage is to model the aggregate traffic produced by the individual source maps with a single "equivalent" map which preserves the traffic load and $H$. The parameterisation of this "equivalent" map is reported in Samuel (1997a and 1997b).

\section{THE MAPS CONTROL PARADIGM}

In the section 3 we have mention how chaotic maps can be used to provide aggregate models for self-similar traffic. We considered the case of aggregation at 
a single node (Samuel, 1997a). A natural extension of nodal models is to couple them together to form networks and then attempt to model and control the characteristics of these networks. In such a model the nodes would be the switching sites and the couplings between the nodes would represent the links between the switching sites. The simplest mathematical models which resemble such a construction are Coupled Map Lattices (CML) where a dynamical system at each node will produce the local traffic input. Coupling will be provided by external input from neighbouring nodes due to the queueing (aggregation) and switching. This is shown schematically in Figure 4.

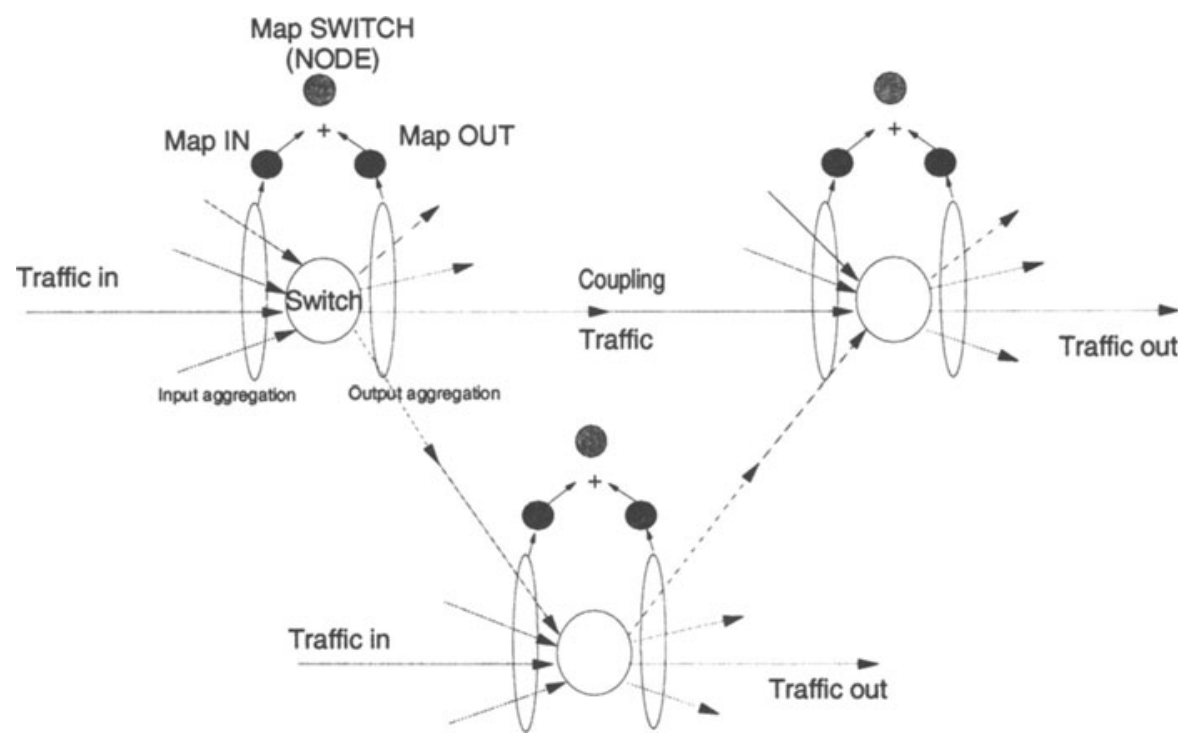

Figure 4 Lattice interpretation of a telecommunications network.

Investigations have been made into regular lattice structures where coupling exists between nodal sites. An important property has been the discovery that global control across all nodes can be obtained via local control at each node (Mondragon, 1997a and 1997b, Arrowsmith, 1996, Ott, 1990).

In these investigations the dynamical behaviour of each nodal site is modelled by a chaotic map and the coupling to the neighbouring nodes imparts perturbations into the orbits of the dynamical system containing the node. Since the chaotic map's orbit possesses the property that any orbit will approach arbitrarily closely every point of the plane described by the chaotic map, then at some point the orbit must take it near to a desired control state. A small feedback control applied at the target point in the orbit places the dynamics of the node into a required state, since the same structure occurs at all nodes. Experimental evidence shows that if desired control state is prescribed for all nodes then eventually the lattice becomes controllable. However, it is possible for neighbouring dynamical behaviour to 
kick a node out of equilibrium via the coupling and so "occasional feedback control" is introduced where the feedback control is activated within the control region around the desired equilibrium for only part of the allowable time, (Mondragon 1997a).

The outline given in section 3, see (Samuel, 1997a) describes how each node site of the lattice structure can be modelled by a controlling map. The individual traffic streams entering a node can be modelled by chaotic maps and in certain models the maps can be aggregated into a single map which describes the behaviour of the traffic at the node. The next step is to provide on-line information on the Hurst parameter; this is necessary to enable the construction of an active dynamic control environment.

We propose a control scheme that actively manipulates the value of $H$. Our intended approach is based in manipulating $H$ via the mean, peakedness and LRD of the traffic stream characteristics via with a local control strategy. The "philosophy" is not to destroy the chaotic behaviour of the traffic but instead to use its variability as a method of control. Chaotic systems are everywhere unstable and thus a small change in the system at any instant produces a large change at later times (this is known as "sensitive dependence on initial conditions" or, more colloquially, as "the butterfly effect") giving the controller "agility" to changes in the traffic over many timescales. Moreover as was noted in the previous section, successful control of coupled chaotic systems can be instigated with local control of each system. The implication of this to networks should be significant, since it suggests that for relatively small control actions applied locally the congestion/buffer occupancy on local and remote switches (relative to the control site) should be reduced. It is envisaged that the use of ATM mechanisms such as $A B R$ via a chaotically initiated control sequence would provide a mechanism for the reduction of $H$ and subsequent control of congestion.

We are proposing two different mechanisms to control traffic that is already in the network. The first mechanism seeks to reduce the variability of the traffic in a specific channel. This can be done by "careful" introduction of empty cells. The control mechanism would modify but not destroy the highly variable behaviour of the traffic. We conjecture that, individually, each of these controlled channels would change very little but that these changes would have a larger effect when the traffic is aggregated in the queue.

The second method is based in a random selector of calls in a node. The random selector would choose which call to admit by weighting dynamically the statistics of the traffic variability. The selector, modelled by a chaotic map, would assign larger probabilities to some channels than others but all the channels would have positive probabilities to be served. The first control technique is termed "Procession" and the second "Order". Conceptual views of the proposed chaotic control regime can be found in Figure. 5. 


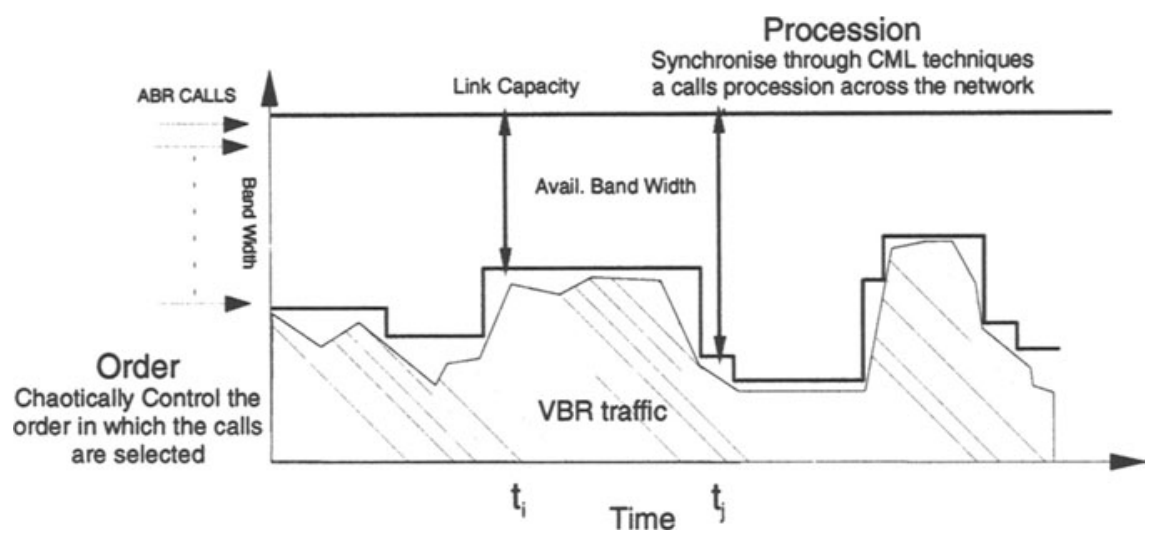

Figure 5 Conceptual view of chaotic network control as applied to ABR.

These two methods of control will be developed using chaotic maps as models of self-similar traffic because it is known that they have the correct characteristics and, moreover, that they can model high traffic rates efficiently (Samuel, 1997a and 1997b).

\section{CONCLUSION}

In this paper we have discussed the problems presented by self-similarity in traffic flow and summarised current views on resolving the problem of self-similar traffic within ATM networks. The weakness of conventional approaches lies in their addressing control over single timescales. We have presented results on the simulation of self-similar traffic by chaotic maps which show long range dependence varying with the map parameters. We have set out a new approach, the MAPS control paradigm, which exploits the dynamical characteristics of chaotic maps in the context of coupled map lattices to effect control over many timescales.

\section{Acknowledgements}

LGS would like to thank NORTEL and EPSRC, RJM would like to thank EPSRC and its Applied Mathematics Initiative for its support during the preparation of this work. 


\section{REFERENCES}

Arrowsmith, D.K., Lansbury, A.N., and Mondragon, R.J. (1996) Control of Arnold circle map. Int. Jour of Bif and Chaos 6:437-453.

Chen, T. and Liu, S. (1995) ATM Switching Systems, Artech House, Boston.

de Prycker, M. (1991) Asynchronous Transfer Mode: Solution for Braodband ISDN. Ellis Horwood, London.

Duffield, N.G., Lewis, J.T., O'Connell, N., Russell, R. and Toomey, F. (1995) Entropy of ATM Traffic Streams: A Tool for Estimating QoS parameters, IEEE Jour. on Sel. Areas in Comm., Vol. 13, No 6, 980-990.

Erramilli, A. Singh, R.P. and Pruthi, P. (1994a) Chaotic Maps as Models of Packet Traffic. ITC 14, 329-338.

Erramilli, A. Pruthi, P., and Willinger, W. (1994b) Modelling Packet Traffic with Chaotic Maps. ISRN KTH/IT/R-94/18-SE, Stockholm-Kista, Sweden.

Erramilli, A. Singh, R.P. and Pruthi, P. (1995) An application of deterministic chaotic maps to model packet traffic. Queueing Systems. Vol 20, 171-206.

Erramilli, A., Naranyan, O. and Willinger W. (1996) Experimental Queueing Analysis with Long-range Dependent Packet Traffic. IEEE/ACM Trans on Networking, Vol 4, No 2, 209-223.

Fan, Z. and Mars, P., (1997) The Impact of the Hurst Parameter and its Crossover effect on Long Range Dependent Traffic Engineering, IEE 14th UKTS, 10/1$10 / 8$.

Fowler H.J. and Leland W.E. (1991) Local Area Network Traffic Characteristics, with Implications for Broadband Network Congestion Management. IEEE Jour. on Sel. Areas in Comm., Vol 9, No 7, 1139-1149.

Krishnan, K.R. (1996) A new class of performance results for a fractional Brownian traffic model, Queueing systems 22, 277-285.

Leland,W.E., Taqqu, M.S., Willinger, W., and Wilson, D. (1994) On the SelfSimilar Nature of Ethernet Traffic (Extended Version). IEEE/ACM Trans on Networking, Vol 2, No 1, 1-15.

Mandelbrot, B. and Van Ness, J.W. (1968) Fractional Brownian Motions, Fractional Noises and Applications. SIAM Review, Vol. 10, No 4, 422-437.

Molnár, S. and Vidács, A. (1997) On Modelling and Shaping Self-similar ATM Traffic, in Vol 2bProc. ITC15, "Teletraffic Contributions for the information Age" (eds. V Ramaswami and P.E. Wirth), Elseveier, 1409-1420.

Mondragon, R.J., and Arrowsmith, D.K. (1997a) Tracking unstable fixed points in parametrically dynamic systems. Phys. Lett. A, Vol.229, No.2.

Mondragon, R.J., and Arrowsmith, D.K., (1997b) On Control of Coupled Map Lattices: Using local dynamics to predict controllability. Int. Jour of Bif and Chaos. 7:No 2, 383-399.

Norros, I. (1993) Studies on a model for connectionless traffic, based on fractional Brownian motion. Conf. On Applied Probability in Engineering, Computer and Communication Sciences, Paris. 
Ott, E., Grebogi, C., and Yorke, J. (1990) Controlling Chaos, Phys. Rev. Lett. 64 (11) 1196-1199, 1990.

Pruthi, P. (1995a) An Application of Chaotic Maps to Packet Traffic Modeling, PhD Dissertation, Royal Institute of Technology, Sweden, ISRN KTH/IT/R-95/19--SE.

Pruthi, P. and Erramilli, A. (1995b) Heavy-Tailed ON/OFF Source Behaviour and Self-Similar Traffic. Proc ICC 95.

Saito, H. (1994) Teletraffic Technologies in ATM Networks. Artech House, Boston. Samuel, L.G., Pitts, J.M., and Mondragón, R.J. (1997a) Towards the Control of Communication Networks by Chaotic Maps: Source Aggregation, in Vol $2 b$ Proc. ITC15, "Teletraffic Contributions for the information Age" (eds. V Ramaswami and P.E. Wirth), Elseveier, 1369-1378.

Samuel, L.G., Pitts, J.M., and Mondragón, R.J., (1997b) Fast Self-similar Traffic Generation, 14th UKTS. 8/1-8/4.

Schormans, J.A., Scharf, E.M. and Pitts, J.M. (1993) Waiting time probabilities in a statistical multiplexer with priorities, IEE Proc. I, vol.140, No. 4, 301-307.

Schormans, J., Azmoodeh, M., Gordhan, S. and Davison, R. (1997) Buffer Partitioning Formula for Different Service Classes of ATM Traffic, submitted to IEE Proc. Comms.

Willinger, W., Taqqu, M.S., Sherman, R., Wilson, D.V. (1997) Self-Similarity through high-variability: statistical analysis of ethernet LAN traffic at the source level. IEEE/ACM Transactions on networking, Vol. 5, No. 1, 71-86. 